\title{
Influência do óleo de semente de uva na degradação termo-oxidativa do polipropileno (PP) reciclado
}

\author{
Influence of grape seed oil into recycled polypropylene \\ (PP) thermo-oxidative degradation
}

\author{
Helson Moreira da Costa ${ }^{1,2}$, Valéria Dutra Ramos ${ }^{2}$, \\ Jacques Satler Andrade Estanislau Cyrino ${ }^{1}$
}

\footnotetext{
${ }^{1}$ Departamento de Materiais (DEMAT), Instituto Politécnico (IPRJ), Universidade do Estado do Rio de Janeiro (UERJ), Rua Hormindo Silva, 25, Lagoinha, Cep: 28625-570, Nova Friburgo, RJ, Brasil.

${ }^{2}$ Universidade Estácio de Sá (UNESA), Rua José Acurcio Benigno, 116, Braunes, Cep: 28611-135, Nova Friburgo, RJ, Brasil.

e-mail:moreirahelson@gmail.com; valdutraramos@gmail.com; jacquessatler@ hotmail.com
}

\section{RESUMO}

O potencial antioxidante do óleo de semente de uva (OSU) foi avaliado após sua adição ao polipropileno reciclado (PP rec.). OSU foi incorporado ao polipropileno reciclado (PP rec.) em teores variáveis de 0,5, 1,0 e 1,5 phr (partes por cem partes de PP). O processamento foi conduzido em uma extrusora de rosca dupla e as diferentes amostras, após serem granuladas, foram submetidas à degradação termo-oxidativa através de termogravimetria (TGA). Os dados experimentais foram tratados por meio de uma metodologia empírica que conjugou modelos cinéticos, presentes na literatura, e ferramentas estatísticas como, por exemplo, a análise de variância (ANOVA). Os resultados da cinética de degradação termo-oxidativa permitiram concluir que OSU apresenta atividade antioxidante efetiva apenas quando o teor adicionado não excede a 0,5 phr e baixas taxas de aquecimento $\left(3^{\circ} \mathrm{C} / \mathrm{min}\right)$ são consideradas.

Palavras-chave: Polipropileno. Óleo de semente de uva. Análise térmica. Termo-oxidação. Cinética.

\section{ABSTRACT}

The antioxidant potential of grape seed oil (OSU) was evaluated after addition into recycled polypropylene (PP rec.). OSU was incorporated into PP rec. in variable contents of $0.5,1.0$ and $1.5 \mathrm{phr}$ (per hundred resin). The processing was carried out in a twin-screw extruder and the different samples, after being granulated, were submitted to thermo-oxidative degradation by thermogravimetry (TGA). Experimental data were treated using an empirical methodology that combined kinetic models, present in the literature, and statistical tools such as analysis of variance (ANOVA). The results of thermo-oxidative degradation kinetics allowed us to conclude that OSU shows effective antioxidant activity only when the added content does not exceed $0.5 \mathrm{phr}$ and low heating rates $\left(3^{\circ} \mathrm{C} / \mathrm{min}\right)$ are considered.

Keywords: Polypropylene. Grape seed oil. Thermal analysis. Thermo-oxidation. Kinetics.

\section{INTRODUÇÃO}

De acordo com DE PAOLI [1] "degradação é qualquer reação química que altera a qualidade de interesse de um material polimérico ou de um composto polimérico". As propriedades de um material polimérico se modificam no decorrer do tempo em função dos vários tipos de ataques físicos e/ou químicos a que o material está sujeito durante o processamento ou uso final dos artefatos. 
O polipropileno é um termoplástico que apresenta como pontos fortes superioridade em transparência, brilho, resistência e acabamento superficial. Além disso, a sustentabilidade também é um aspecto positivo em relação aos demais polímeros, tendo em vista que o polipropileno é 100\% reciclável e apresenta baixa emissão de gases de efeito estufa na atmosfera durante sua produção e transporte. Porém, a alta versatilidade de aplicações esbarra em uma estrutura química onde a existência de um átomo de hidrogênio terciário muito reativo traz uma alta probabilidade de transferência de cadeia intramolecular; e uma velocidade de oxidação dos radicais alquila proporcional às velocidades de outras reações competitivas [2].

O uso de aditivos estabilizantes em polímeros para prevenir o "envelhecimento" (degradação) é tão antiga quanto a descoberta destes materiais [2]. A análise da atividade antioxidante de compostos naturais teve início em 1952 com as especiarias, ingredientes utilizados nos alimentos desde os primórdios da história, não somente para melhorar ou ressaltar suas características sensoriais, mas também para preservá-los. Atualmente, várias fontes de antioxidantes naturais são conhecidas e algumas são amplamente encontradas no reino vegetal, pois em todas as plantas podem ser encontrados princípios ativos importantes, sintetizados pelo metabolismo secundário, que dão origem a uma série de substâncias conhecidas como alcaloides, flavonoides, cumarinas, saporinas, taninos, óleos essenciais, entre outras. Genericamente, os antioxidantes podem ser divididos em duas classes: antioxidantes com atividade enzimática e os antioxidantes sem essa atividade. Na primeira, estão os compostos capazes de bloquear a iniciação da oxidação, ou seja, as enzimas que removem as espécies reativas ao oxigênio. Na segunda classe, estão moléculas que interagem com as espécies radicalares e são consumidas durante a reação. Nesta classificação, incluem-se os antioxidantes naturais e sintéticos como, por exemplo, os compostos fenólicos [3 - 6].

OLIVEIRA e colaboradores [6], por exemplo, ressaltam a importância do uso de antioxidantes naturais em produtos cárneos. De acordo com os autores, a utilização de antioxidantes sintéticos - butil-hidroxi-anisol (BHA), butil-hidroxi-tolueno (BHT), terc-butil-hidroxiquinona (TBHQ) e propil-galato (PG) - vem sendo reduzida em função de pesquisas intensificadas a partir de 1980, as quais comprovam a toxicidade de tais substâncias, e do aumento da consciência dos consumidores no que diz respeito à segurança dos aditivos alimentares. FRITSCH e colaboradores [7] chamam a atenção para o enorme crescimento da investigação das propriedades antioxidantes de espécies vegetais e destacam que quase todas as plantas e os micro-organismos contêm antioxidantes de vários tipos, que por várias razões (disponibilidade, segurança alimentar, econômica) podem ser usados como uma fonte natural. Em seu trabalho, os pesquisadores avaliaram a atividade antioxidante de espécies obtidas na região sul do Brasil tais como a amora (Morus nigra), a pitanga (Eugenia uniflora), o araçá (Psidium cattleianum), a guabirova (Camponesia guaviroba), o butiá-yataí (Butia yatay), a cereja-do-rio-grande (Psidium cattleianum) e o vacum (Allophylus edulis).

OLIVEIRA [8] demonstra preocupação com a grande quantidade de resíduos gerados pelo aumento da produção de alimentos a partir de frutas e vegetais. De acordo com a pesquisa, as frutas e os legumes são fontes de compostos bioativos como polifenóis, vitamina $\mathrm{C}$, carotenoides e tocoferol que apresentam efeitos benéficos para a saúde por atuarem na prevenção de doenças. Tal como as frutas, os resíduos apresentam esses compostos bioativos evidenciando potencial de aproveitamento pela indústria para o desenvolvimento de novos produtos, além de levar à redução dos custos financeiros para o manejo e descarte adequado. Os resíduos obtidos do processamento de frutas podem ainda apresentar substâncias nutritivas, como óleo essencial, proteínas, enzimas, metabólitos secundários e lipídeos.

A uva é fonte de diversos compostos fenólicos em elevadas concentrações e os subprodutos da vinificação, em sua maioria, podem manter quantidades apreciáveis dessas substâncias como os flavonoides (antocianinas, flavanóis e flavonóis), os estilbenos (resveratrol), os ácidos fenólicos (derivados dos ácidos hidroxicinâmicos e hidroxibenzóicos) e uma larga variedade de taninos. Os ácidos fenólicos são encontrados em maior quantidade nos tecidos da polpa da uva ( 80 a $85 \%$ ), sendo que sua concentração diminui com o amadurecimento do fruto e varia de acordo com o cultivo [9, 10]. ZOPELLARO e colaboradores [11], por exemplo, apontam que a farinha do resíduo da uva é excelente fonte de compostos fenólicos, possuindo um bom potencial antioxidante e constituindo uma alternativa para a diminuição das perdas econômicas dentro de uma empresa vinícola, além da redução do impacto ambiental dos resíduos da atividade.

$\mathrm{Na}$ literatura não são encontrados trabalhos específicos sobre o efeito de óleos vegetais, em particular, o óleo de semente de uva, como possíveis aditivos antioxidantes em termoplásticos. Desta forma, em investigações inéditas e preliminares [12 - 14], nosso grupo de pesquisa procurou avaliar a influência de óleos vegetais comerciais - óleo de mamona, óleo de castanha do Brasil e óleo de semente de uva - na degradação termo-oxidativa e termomecânica combinadas do polipropileno (PP) reciclado. Em tais pesquisas, um efeito antioxidante promissor para o óleo de semente de uva foi detectado. Agora, nesta investigação, o objetivo principal foi determinar quais as condições mais adequadas que potencializam o óleo de semente uva como aditivo antioxidante para o polipropileno reciclado. Para tanto, técnicas estatísticas (ANOVA e teste t) em 
conjunto com uma metodologia empírica, desenvolvida em trabalhos prévios [12 - 14], foram usadas. Análise térmica, termogravimetria (TGA/DTG), foi empregada para que a degradação termo-oxidativa do polipropileno pudesse ter sua cinética avaliada, assim como a energia de ativação e outros parâmetros de interesse pudessem ser determinados.

\section{MATERIAIS E MÉTODOS}

O polipropileno (PP) reciclado comercial, PPH.210.40.T, foi comprado junto à empresa Plastimil Inovações em Compostos e Reciclados, Vinhedo, SP. De acordo com informações do fornecedor, a família PPH é composta por termoplásticos formulados através da polimerização do propeno, apresentando melhor rigidez em relação aos copolímeros. Além disso, são resistentes a altas temperaturas e dispõem de boa resistência química contra ácidos, bases e solventes. O PPH.210.40.T apresenta coloração preta em função da presença $40 \%$, em massa, de negro de fumo e é fornecido em grãos, sendo adequado para a obtenção de peças automotivas através do processamento por injeção. As propriedades são apresentadas na Tabela 1.

Tabela 1: Propriedades do termoplástico PPH.210.40.T

\begin{tabular}{l|c|r}
\hline \multicolumn{1}{c|}{ PROPRIEDADE } & VALOR & MÉTODO \\
\hline Índice de fluidez & $12 \mathrm{~g} / 10 \mathrm{~min}$ & ASTM D 1238; 230 ${ }^{\circ} \mathrm{C} ; 2,16 \mathrm{~kg}$ \\
\hline Densidade & $1,2 \mathrm{~g} / \mathrm{cm}^{3}$ & ASTM D 792 \\
\hline Teor de carga & $40 \%$ & FT-IT-18; 670 ${ }^{\circ} \mathrm{C} ; 40 \mathrm{~min}$ \\
\hline Contração & $0,7 \%$ & FT-IT-45 \\
\hline Envelhecimento & $>700 \mathrm{~h}$ & Estufa; $150^{\circ} \mathrm{C}$ \\
\hline Impacto & $30 \mathrm{~J} / \mathrm{m}$ & Izod; ASTM D 256; 23 ${ }^{\circ} \mathrm{C}$, com entalhe \\
\hline Resistência à tração & $25 \mathrm{MPa}$ & ASTM D 638 \\
\hline
\end{tabular}

O óleo de semente de uva, designado neste artigo pela sigla OSU, foi comprado junto à empresa Quinarí, localizada em Ponta Grossa, Paraná. OSU é um dos óleos mais concentrados em ácidos graxos poliinsaturados, representando cerca de $95 \%$ de sua composição. Além de rico em insaturados, ele apresenta propriedades bastante interessantes do ponto de vista cosmético e alimentício, sendo rico em vitamina E e proantocianidinas, importantes agentes antioxidantes [5]. O óleo vegetal foi usado como recebido, sem quaisquer tipos de tratamentos físico-químicos.

A amostra de polipropileno reciclado (PP rec.) e as composições de PP rec. contendo OSU em diferentes teores, após pesagem das concentrações pré-determinadas (ver Tabela 2), foram processadas em triplicata em extrusora de dupla rosca da marca Leistritz ${ }^{\circledR}$, modelo ZSE 18 Maxx. A extrusora estava equipada com roscas de $19 \mathrm{~mm}$ e uma taxa de produção de $10 \mathrm{~kg} / \mathrm{h}$ foi selecionada para o processamento. As condições de extrusão escolhidas foram: velocidade de rotação igual a $100 \mathrm{rpm}$; e, faixa de temperatura de $180^{\circ} \mathrm{C}$ (zona de alimentação) até $220^{\circ} \mathrm{C}$ (zona da matriz). As amostras processadas, em forma de três fios contínuos (matriz macarrão), foram resfriadas em água em temperatura ambiente e, posteriormente, granuladas em moinho de facas. A secagem do material granulado ocorreu em estufa com circulação de ar por $24 \mathrm{~h}$ e temperatura de $80^{\circ} \mathrm{C}$.

Tabela 2: Composições de PP rec./OSU processadas em extrusora de dupla rosca

\begin{tabular}{l|c}
\hline \multicolumn{1}{c|}{ MISTURA } & OSU (phr) \\
\hline PP rec. & 0,0 \\
\hline PP rec./OSU & 0,5 \\
& 1,0 \\
& 1,5 \\
\hline
\end{tabular}

"As quantidades do óleo vegetal estão referenciadas em phr, ou seja, partes por cem partes de resina. Assim, por exemplo, PP rec./OSU 0,5 phr corresponde a 0,5 grama de OSU adicionado para cada $100 \mathrm{~g}$ de PP rec.

A análise térmica foi conduzida em um equipamento da marca Perkin Elmer, modelo STA-6000 Silmutaneous Thermal Analyzer. Os termogramas gerados pelas análises de termogravimetria (TGA/DTG) foram interpretados com auxílio do programa Pyris Thermal Analysis ${ }^{\mathrm{TM}} \AA$, versão 10.1, instalado no próprio equi- 
pamento. As condições escolhidas para promover a degradação termo-oxidativa via análises termogravimétricas em triplicatas foram: atmosfera oxidativa (ar sintético), com fluxo de ar de $20 \mathrm{~mL} / \mathrm{min}$; taxas de aquecimento de $3^{\circ} \mathrm{C} / \mathrm{min}, 6^{\circ} \mathrm{C} / \mathrm{min}$ e $12^{\circ} \mathrm{C} / \mathrm{min}$ nos estudos de cinética de degradação; o material do cadinho ou porta amostra foi porcelana; quantidade de amostra em cada análise entre 10 e $15 \mathrm{mg}$; e faixa de varredura de temperatura de $30^{\circ} \mathrm{C}$ até $600^{\circ} \mathrm{C}$.

Os dados extraídos dos termogramas permitiram a determinação do hipotético mecanismo cinético para a degradação do polipropileno reciclado puro ou contendo os diferentes teores de OSU. De igual maneira, parâmetros como a energia de ativação e a constante pré-exponencial também foram estimados. Em todas as etapas do tratamento de dados, o programa SPSS 12.0 $0_{\circledR}^{\mathrm{TM}_{\circledast}}$ foi utilizado. A metodologia para o tratamento dos dados experimentais é descrita nas etapas a seguir.

\subsection{Modelagem da cinética de degradação e análise estatística}

\section{- Etapa 1: Determinação dos valores de $E_{i s o}$}

Para a determinação dos valores da energia de ativação isoconversional $\left(\mathrm{E}_{\mathrm{iso}}\right)$, o método selecionado foi o método de Ozawa-Flynn-Wall (método OFW), o qual emprega uma aproximação numérica para a resolução da integral de temperatura de Arrhenius. Usando a aproximação mais simples, sugerida por Doyle, temos [15 - 17]:

$$
\ln \left(\beta_{i}\right)=\ln \frac{A_{x} E_{x_{i}}}{R g_{i}(x)}-5,331-1,052 \frac{E_{x_{i}}}{R T_{x, i}}
$$

Onde: i corresponde a uma taxa de aquecimento em particular; $\mathrm{R}$ é a constante universal dos gases perfeitos; $A_{x_{i}}$ e $E_{x_{i}}$ correspondem à constante pré-exponencial e à energia de ativação, respectivamente; $T_{x, i}$ é a temperatura para certa porcentagem $x$ de degradação do material em uma taxa de aquecimento $\beta_{i}$; e, $g_{i}(x)$ é um modelo cinético hipotético (ver Tabela 3).

De acordo com esse método, a energia de ativação pode ser calculada, para certa conversão $x$, a partir da inclinação da linha reta gerada pelo gráfico $\ln \left(\beta_{\mathrm{i}}\right)$ vs. $1 / \mathrm{T}_{\mathrm{x}, \mathrm{i}}$. É importante ressaltar que esse método permite a estimativa de $\mathrm{E}_{\mathrm{x}_{\mathrm{i}}}$ sem o conhecimento de qualquer função $\mathrm{g}_{\mathrm{i}}(\mathrm{x})$ específica para a cinética. $\mathrm{O}$ método OFW assume que $\mathrm{E}_{\mathrm{x}_{\mathrm{i}}}$ é constante, então, um erro sistemático na estimativa do parâmetro deve ser esperado, se este variar com $x[15-17]$.

Com os valores de $\mathrm{E}_{\mathrm{x}_{\mathrm{i}}}$ encontrados para cada grau de conversão $x$, a metodologia estatística denominada teste $t$ para uma amostra foi aplicada para a determinação do intervalo de confiança para o valor médio da energia de ativação do processo de degradação. As hipóteses para a aplicação da metodologia foram: (i) os dados são contínuos (não discretos); (ii) os dados seguem uma distribuição de probabilidades normal; e (iii) a amostra é resultado de uma amostragem aleatória a partir da população.

\section{- Etapa 2: Modelos de ajuste e peneiramento (seleção dos modelos cinéticos $g(x)$ )}

Nesta etapa foi utilizado um dos mais populares modelos de ajuste, o método de Coasts-Redfern (CR). Esse método é baseado na Equação 2 [17 - 20]:

$$
\ln \left(\frac{g_{i}(x)}{T_{x_{i}}^{2}}\right) \cong \ln \left(\frac{A_{i} R}{\beta E_{a_{i}}}\right)-\frac{E_{a_{i}}}{R T_{x_{i}}}
$$

A escolha de um modelo cinético $\mathrm{g}(\mathrm{x})$ adequado permite que o gráfico de $\ln \left(\mathrm{g}_{\mathrm{i}}(\mathrm{x}) / \mathrm{T}_{\mathrm{x}_{\mathrm{i}}}^{2}\right)$ versus $1 / \mathrm{T}_{\mathrm{x}_{\mathrm{i}}}$ dê origem a uma linha reta, cuja inclinação e o coeficiente linear geram uma estimativa da energia de ativação $\left(\mathrm{E}_{\mathrm{a}_{\mathrm{i}}}\right)$ e do parâmetro $\mathrm{A}_{\mathrm{i}}$, respectivamente. Diversos modelos cinéticos $\mathrm{g}_{\mathrm{i}}(\mathrm{x})$, ver Tabela 1 , foram empregados e diversos valores de $\mathrm{E}_{\mathrm{a}}$ e A foram obtidos. A etapa denominada peneiramento foi, então, implementada considerando-se a análise do coeficiente de correlação, obtido pela Equação 2; e, a comparação entre os valores obtidos de $\mathrm{E}_{\mathrm{a}}$ e o intervalo de confiança para $\mathrm{E}_{\mathrm{a}}$ determinado na Etapa 1.

- Etapa 3: Determinação do fator pré-exponencial e da energia de ativação do processo de degradação termo-oxidativa usando o método dos parâmetros cinéticos invariantes (IKP)

No método IKP, o "verdadeiro" modelo cinético pode ser obtido através do cálculo de uma série de valores de $\ln \mathrm{A}$ e $\mathrm{E}_{\mathrm{a}}$, em diferentes taxas de aquecimento. Se o efeito de compensação entre ln $\mathrm{A}$ e $\mathrm{E}_{\mathrm{a}}$ existir, 
então, gráficos lineares de ln A vs. $\mathrm{E}_{\mathrm{a}}$ devem ser obtidos em cada taxa de aquecimento, de acordo com a Equação 3 [15 - 17]:

$$
\ln A=a^{*}+b^{*} E_{a}
$$

As retas geradas pela Equação 3 devem apresentar interseção em um ponto, o qual corresponde aos valores "reais" de $E_{a}$ e ln A - parâmetros cinéticos invariantes de Lesnikovich e Levchik $\left(\mathrm{E}_{\text {inv }}\right.$ e $\left.A_{\text {inv }}\right)$. Uma vez que certas variações nas condições experimentais exercem influência nas regiões de interseção, o ponto de interseção é somente uma aproximação. Assim sendo, a fim de se minimizar o efeito de tais variações, a Equação 4 pode ser aplicada [15 - 17]:

$$
a^{*}=\ln A_{i n v}-b^{*} E_{\text {inv }}
$$

A Equação 4 é chamada de supercorrelação e deve ser observado que o método IKP pode ser usado somente se $\mathrm{E}_{\mathrm{a}}$ não depende da conversão $\mathrm{x}$, um pré-requisito que pode ser verificado por métodos isoconversionais [15 - 17].

Tabela 3: Expressões mais comuns para as funções $g(x)$ nos mecanismos de reação em estado sólido [16, 1820].

\begin{tabular}{cccc}
\hline $\mathbf{N}^{\mathbf{O}}$ & MOD. & $\mathbf{g}(\mathbf{x})$ & MECANISMO DETERMINANTE DA TAXA \\
\hline & & PROCESSO QUÍMICO (ORDEM DE REAÇÃO) \\
\hline $\mathbf{1}$ & $\mathrm{F}_{1 / 3}$ & $1-(1-\mathrm{x})^{2 / 3}$ & Reação química \\
$\mathbf{2}$ & $\mathrm{F}_{3 / 4}$ & $1-(1-\mathrm{x})^{1 / 4}$ & Reação química \\
$\mathbf{3}$ & $\mathrm{F}_{3 / 2}$ & $(1-\mathrm{x})^{-1 / 2}-1$ & Reação química \\
$\mathbf{4}$ & $\mathrm{F}_{2}$ & $(1-\mathrm{x})^{-1}-1$ & Reação química \\
$\mathbf{5}$ & $\mathrm{F}_{3}$ & $(1-\mathrm{x})^{-2}-1$ & Reação química \\
$\mathbf{6}$ & $\mathrm{F}_{4}$ & $(1-\mathrm{x})^{-3}-1$ & Reação química \\
$\mathbf{7}$ & $\mathrm{G}_{1}$ & $1-(1-\mathrm{x})^{2}$ & Reação química \\
$\mathbf{8}$ & $\mathrm{G}_{2}$ & $1-(1-\mathrm{x})^{3}$ & Reação química \\
$\mathbf{9}$ & $\mathrm{G}_{3}$ & $1-(1-\mathrm{x})^{4}$ & Reação química \\
\hline
\end{tabular}

\section{EQUAÇÕES COM TAXA ACELERADA}

\begin{tabular}{cccc}
\hline $\mathbf{1 0}$ & $\mathrm{P}_{3 / 2}$ & $\mathrm{x}^{3 / 2}$ & Nucleação (lei das potências) \\
$\mathbf{1 1}$ & $\mathrm{P}_{1 / 2}$ & $\mathrm{x}^{1 / 2}$ & Nucleação (lei das potências) \\
$\mathbf{1 2}$ & $\mathrm{P}_{1 / 3}$ & $\mathrm{x}^{1 / 3}$ & Nucleação (lei das potências) \\
$\mathbf{1 3}$ & $\mathrm{P}_{1 / 4}$ & $\mathrm{x}^{1 / 4}$ & Nucleação (lei das potências) \\
$\mathbf{1 4}$ & $\mathrm{P}_{2}$ & $\mathrm{x}^{2}$ & Nucleação (lei parabólica) \\
$\mathbf{1 5}$ & $\mathrm{E}_{1}$ & $\ln (\mathrm{x})$ & Nucleação (lei exponencial) \\
$\mathbf{1 6}$ & $\mathrm{E}_{2}$ & $\ln \left(\mathrm{x}^{2}\right)$ & Nucleação (lei exponencial) \\
\hline
\end{tabular}

\section{EQUAÇÕES COM TAXA SIGMOIDAL (NUCLEAÇÃO E CRESCIMENTO DE NÚCLEOS)}

\begin{tabular}{cccc}
\hline $\mathbf{1 7}$ & $\mathrm{A}_{1}$ & $-\ln (1-\mathrm{x})$ & Nucleação randômica (Mampel) \\
$\mathbf{1 8}$ & $\mathrm{A}_{2 / 3}$ & {$[-\ln (1-\mathrm{x})]^{3 / 2}$} & Nucleação randômica (Avrami-Erofeev) \\
$\mathbf{1 9}$ & $\mathrm{A}_{3 / 2}$ & {$[-\ln (1-\mathrm{x})]^{2 / 3}$} & Nucleação randômica (Avrami-Erofeev) \\
$\mathbf{2 0}$ & $\mathrm{A}_{3 / 4}$ & {$[-\ln (1-\mathrm{x})]^{4 / 3}$} & Nucleação randômica (Avrami-Erofeev) \\
$\mathbf{2 1}$ & $\mathrm{A}_{5 / 2}$ & {$[-\ln (1-\mathrm{x})]^{2 / 5}$} & Nucleação randômica (Avrami-Erofeev) \\
$\mathbf{2 2}$ & $\mathrm{A}_{2}$ & {$[-\ln (1-\mathrm{x})]^{1 / 2}$} & Nucleação randômica (Avrami-Erofeev) \\
$\mathbf{2 3}$ & $\mathrm{A}_{3}$ & {$[-\ln (1-\mathrm{x})]^{1 / 3}$} & Nucleação randômica (Avrami-Erofeev) \\
$\mathbf{2 4}$ & $\mathrm{A}_{4}$ & {$[-\ln (1-\mathrm{x})]^{1 / 4}$} & Nucleação randômica (Avrami-Erofeev) \\
$\mathbf{2 5}$ & $\mathrm{A}_{1 / 2}$ & {$[-\ln (1-\mathrm{x})]^{2}$} & Nucleação randômica (Avrami-Erofeev) \\
$\mathbf{2 6}$ & $\mathrm{A}_{1 / 3}$ & {$[-\ln (1-\mathrm{x})]^{3}$} & Nucleação randômica (Avrami-Erofeev) \\
$\mathbf{2 7}$ & $\mathrm{A}_{1 / 4}$ & {$[-\ln (1-\mathrm{x})]^{4}$} & Nucleação randômica (Avrami-Erofeev) \\
$\mathbf{2 8}$ & $\mathrm{B}_{1}$ & $\ln [\mathrm{x} /(1-\mathrm{x})]$ & Núcleos de ramificação (Prout-Tompkins) \\
\hline
\end{tabular}


EQUAÇÕES COM TAXA DESACELERADA (CONTORNO DE FASE RESTRITO)

\begin{tabular}{cccc}
\hline $\mathbf{2 9}$ & $\mathrm{R}_{1}$ & $\mathrm{x}$ & Simetria plana \\
$\mathbf{3 0}$ & $\mathrm{R}_{2}$ & $1-(1-\mathrm{x})^{1 / 2}$ & Simetria cilíndrica \\
S1 & $\mathrm{R}_{3}$ & $1-(1-\mathrm{x})^{1 / 3}$ & Simetria esférica \\
\hline \multicolumn{4}{c}{ EQUAÇÕES COM TAXA DESACELERADA (MECANISMO DE DIFUSÃO) } \\
\hline $\mathbf{3 2}$ & $\mathrm{D}_{1}$ & $\mathrm{x}^{2}$ & Difusão unidimensional \\
$\mathbf{3 3}$ & $\mathrm{D}_{2}$ & $\mathrm{x}+(1-\mathrm{x}) \ln (1-\mathrm{x})$ & Difusão bidimensional \\
$\mathbf{3 4}$ & $\mathrm{D}_{3}$ & {$\left[1-(1-\mathrm{x})^{1 / 3}\right]^{2}$} & Difusão tridimensional (Jander) \\
$\mathbf{3 5}$ & $\mathrm{D}_{4}$ & $1-(2 / 3) \mathrm{x}-(1-\mathrm{x})^{2 / 3}$ & Difusão tridimensional (Ginstling-Brounshtein) \\
$\mathbf{3 6}$ & $\mathrm{D}_{5}$ & {$\left[(1-\mathrm{x})^{-1 / 3}-1\right]^{2}$} & Difusão tridimensional (Crank) \\
$\mathbf{3 7}$ & $\mathrm{D}_{6}$ & {$\left[(1+\mathrm{x})^{1 / 3}-1\right]^{2}$} & Difusão tridimensional (Komatsu) \\
$\mathbf{3 8}$ & $\mathrm{D}_{7}$ & $1+(2 / 3) \mathrm{x}-(1+\mathrm{x})^{2 / 3}$ & Difusão tridimensional \\
$\mathbf{3 9}$ & $\mathrm{D}_{8}$ & {$\left[(1+\mathrm{x})^{-1 / 3}-1\right]^{2}$} & Difusão tridimensional (Zuravlev, Lesbhim e Temelman \\
\hline
\end{tabular}

OUTRAS EQUAÇÕES CINÉTICAS SEM MECANISMO JUSTIFICADO

\begin{tabular}{lll}
\hline $\mathbf{4 0}$ & $\mathrm{G}_{7}$ & {$\left[1-(1-\mathrm{x})^{1 / 2}\right]^{1 / 2}$} \\
$\mathbf{4 1}$ & $\mathrm{G}_{8}$ & {$\left[1-(1-\mathrm{x})^{1 / 3}\right]^{1 / 2}$} \\
\hline
\end{tabular}

Os modelos cinéticos apesentados na Tabela 3 podem ser genericamente classificados segundo o formato do gráfico exibido a partir de curvas isotérmicas ( $x$ vs. T ou d $x / \mathrm{dt}$ vs. $x$ ) ou, ainda, por intermédio de hipóteses sobre o mecanismo [21].

Baseado no formato, os modelos cinéticos podem ser agrupados em: modelos de taxa acelerada $\left(\mathrm{P}_{\mathrm{i}} \mathrm{e}\right.$ $\left.E_{i}\right)$; modelos de taxa desacelerada $\left(R_{i}\right.$ e $\left.D_{i}\right)$; modelos lineares $\left(F_{i}\right.$ e $\left.G_{i}\right)$; e modelos sigmoidais $\left(A_{i}\right)$. Os modelos de taxa acelerada são aqueles onde a taxa da reação $(\mathrm{d} x / \mathrm{dt})$ aumenta, isto é, acelera conforme a reação tem continuidade. De forma análoga, os modelos de taxa desacelerada exibem a taxa de reação diminuindo com o progresso da reação. Os modelos lineares mantêm a taxa de reação constante e são aplicados em processos químicos na forma de modelos de ordem de reação. Finalmente, os modelos sigmoidais apresentam uma relação entre a taxa de reação e $x$ em forma de $S$ [21].

\section{RESULTADOS E DISCUSSÃO}

As diferentes amostras processadas em extrusora de rosca dupla, conforme a Tabela 2, foram submetidas à degradação termo-oxidativa. Os dados da termogravimetria (TGA) e termogravimetria derivativa (DTG) estão resumidos na Tabela 4.

Além dos dados sumarizados na Tabela 4, as curvas experimentais de perda de massa, resultantes das análises termogravimétricas, foram transformadas em um gráfico do tipo boxplot (diagrama de caixa) conforme ilustrado na Figura 1. A caixa branca representa o PP rec. sem adição do óleo vegetal, enquanto as caixas em tons de roxo representam o PP rec. com um teor específico de OSU. Os pontos em preto no interior de cada caixa representam valores em particular de perda de massa; de baixo para cima na caixa, temos $5 \%$, $10 \%, 15 \%, \ldots, 55 \%$ de perda de massa.

A análise dos dados da Tabela 4 e da Figura 1 permitem que algumas observações gerais possam ser obtidas. Inicialmente, pôde ser verificado que todas as composições contendo o óleo de semente de uva (OSU) demonstram um residual de massa, em média, de 35\%. Tal resíduo está associado a presença de negro de fumo no termoplástico reciclado, conforme as informações do fornecedor do PP rec. descritas na Tabela 1. 
Tabela 4: Efeito do óleo semente de uva sobre a degradação termo-oxidativa do PP reciclado

\begin{tabular}{|c|c|c|c|c|c|c|}
\hline Taxa $-3^{\circ} \mathrm{C} / \mathrm{min}$ & $\mathrm{T}_{\text {onset }}\left({ }^{\circ} \mathrm{C}\right)$ & $\mathrm{T}_{\text {endset }}\left({ }^{\circ} \mathrm{C}\right)$ & $\mathrm{T}_{5 \%}\left({ }^{\circ} \mathrm{C}\right)$ & $t_{1 / 2}(s)$ & $\mathrm{T}_{\text {pico }}\left({ }^{\circ} \mathrm{C}\right)$ & $\Delta \mathrm{m}(\%)$ \\
\hline 0,0 phr & 343,5 & 432,5 & 322,3 & 7742,8 & 424,9 & 34,9 \\
\hline $0,5 \mathrm{phr}$ & 325,1 & 433,0 & 325,2 & 7774,7 & 425,0 & 34,6 \\
\hline $1,0 \mathrm{phr}$ & 323,4 & 438,3 & 327,8 & 7837,9 & 429,1 & 34,5 \\
\hline $1,5 \mathrm{phr}$ & 322,1 & 441,9 & 332,1 & 7897,4 & 433,0 & 34,4 \\
\hline \multicolumn{7}{|l|}{ Taxa $-6^{\circ} \mathrm{C} / \mathrm{min}$} \\
\hline 0,0 phr & 363,3 & 448,5 & 351,6 & 4066,4 & 443,8 & 35,1 \\
\hline $0,5 \mathrm{phr}$ & 360,7 & 450,4 & 351,0 & 4057,8 & 441,7 & 35,0 \\
\hline $1,0 \mathrm{phr}$ & 356,8 & 452,4 & 349,9 & 4038,5 & 440,1 & 34,8 \\
\hline $1,5 \mathrm{phr}$ & 358,2 & 453,6 & 349,4 & 4022,6 & 440,0 & 34,6 \\
\hline \multicolumn{7}{|l|}{ Taxa $-12^{\circ} \mathrm{C} / \mathrm{min}$} \\
\hline 0,0 phr & 401,8 & 464,5 & 375,7 & 2159,0 & 464,5 & 35,0 \\
\hline $0,5 \mathrm{phr}$ & 393,1 & 467,9 & 375,3 & 2133,3 & 458,7 & 35,0 \\
\hline $1,0 \mathrm{phr}$ & 382,6 & 469,4 & 373,4 & 2110,8 & 456,6 & 34,9 \\
\hline $1,5 \mathrm{phr}$ & 366,3 & 470,1 & 372,4 & 2087,3 & 454,3 & 34,8 \\
\hline
\end{tabular}

$\mathrm{T}_{\text {onset }}$, temperatura do início do processo de degradação; $\mathrm{T}_{\text {endset }}$, temperatura do término do processo de degradação; $\mathrm{T}_{5 \%}$, temperatura para que a amostra perca $5 \%$ de massa; $\mathrm{t}_{1 / 2}$, tempo de meia-vida para a amostra; $\mathrm{T}_{\text {pico, }}$, temperatura alcançada quando a taxa de perda de massa é máxima; e, $\Delta \mathrm{m} \%$, porcentagem residual da amostra ao fim da análise termogravimétrica.
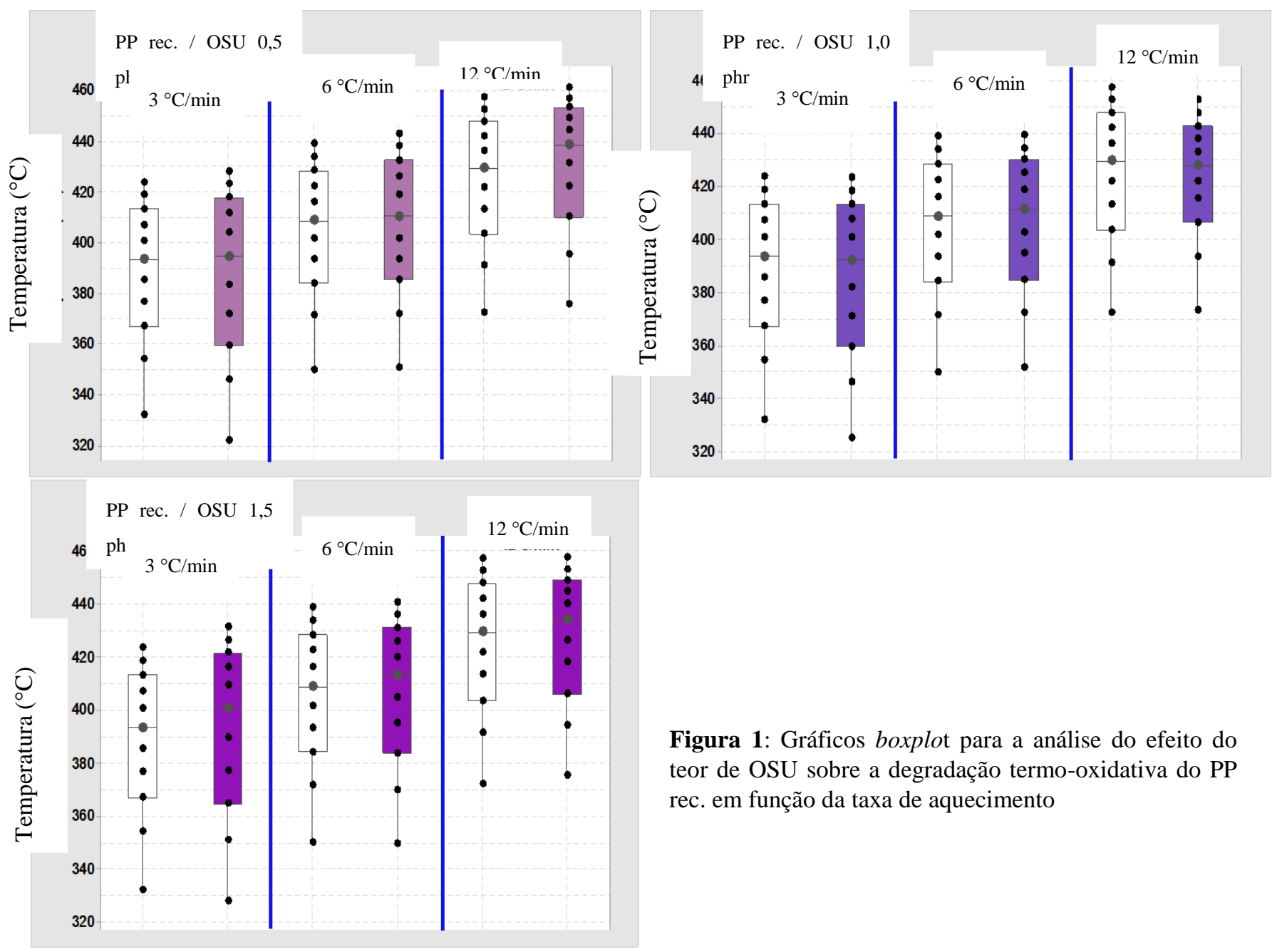

Figura 1: Gráficos boxplot para a análise do efeito do teor de OSU sobre a degradação termo-oxidativa do PP rec. em função da taxa de aquecimento 
Além disso, OSU parece, independentemente do teor adicionado ao PP rec., atuar da mesma maneira quando uma baixa taxa de aquecimento é imposta, o que pode ser percebido pela análise do intervalo interquartílico (IQ), que representa o intervalo de temperatura no qual a perda de massa da amostra situa-se entre $15 \%$ e $45 \%$ ou, em outras palavras, o tamanho da caixa retangular nos gráficos bloxplot. Observa-se que na taxa de aquecimento de $3^{\circ} \mathrm{C} / \mathrm{min}$, composições de PP rec. com OSU exibem um IQ superior ao do PP rec.; por exemplo, para a composição de PP rec./OSU 0,5 phr temos um IQ de 58,5 ${ }^{\circ} \mathrm{C}$, enquanto para o $\mathrm{PP}$ rec. o IQ é de $46,3^{\circ} \mathrm{C}$ (Figura 1).

Podemos especular que OSU age retardando a degradação termo-oxidativa do termoplástico. Os aumentos registrados em parâmetros como no tempo de meia-vida $\left(\mathrm{t}_{1 / 2}\right)$, ou seja, do tempo necessário para que $50 \%$ da amostra inicial seja degradada, e na temperatura de pico $\left(\mathrm{T}_{\text {pico }}\right)$, a qual representa o máximo de taxa de perda de massa (Tabela 4), podem corroborar tal hipótese. Em contrapartida, quando maiores taxas de aquecimento, como $6^{\circ} \mathrm{C} / \mathrm{min}$ e $12^{\circ} \mathrm{C} / \mathrm{min}$, são utilizadas, OSU parece atuar de forma contrária ao efeito esperado, ou seja, o óleo vegetal parece tender a acelerar a degradação do termoplástico. Além de redução do IQ (observe os tamanhos das caixas roxas do boxplot) (Figura 1), é também verificada uma redução de valores de $\mathrm{t}_{1 / 2}$ e $\mathrm{T}_{\text {pico, }}$, particularmente, em composições que usam OSU em 1,0 phr e 1,5 phr.

De acordo com a literatura [6,7], a uva é fonte de diversos compostos fenólicos em elevadas concentrações. Ao mesmo tempo, no processo de aditivação de materiais poliméricos é encontrado que os principais tipos de antioxidantes primários são as aminas e os fenóis, que possuem átomos de hidrogênio mais reativos com os radicais peróxi ou alcoxi do que os átomos de hidrogênio da cadeia do polímero [1, 2]. Consequentemente, podemos teorizar que a presença do óleo vegetal OSU, acima de determinado valor ótimo, proporcione a existência de reações em paralelo e/ou competitivas. Reações que não necessariamente impedem ou retardam o mecanismo de degradação termo-oxidativa do PP rec. Desta maneira, OSU, embora rico em compostos fenólicos, não conseguiria exercer um papel antioxidante ativo quando aplicado em teores acima de $0,5 \mathrm{phr}$.

O implemento do estudo cinético do processo de degradação termo-oxidativa das composições de PP rec. e PP rec./OSU torna-se necessário para uma melhor compreensão dos resultados. Assim sendo, a etapa 1 da metodologia foi posta em execução. Na Figura 2, os valores de energia de ativação, calculados pela Equação 1, para cada amostra em particular são apresentados.

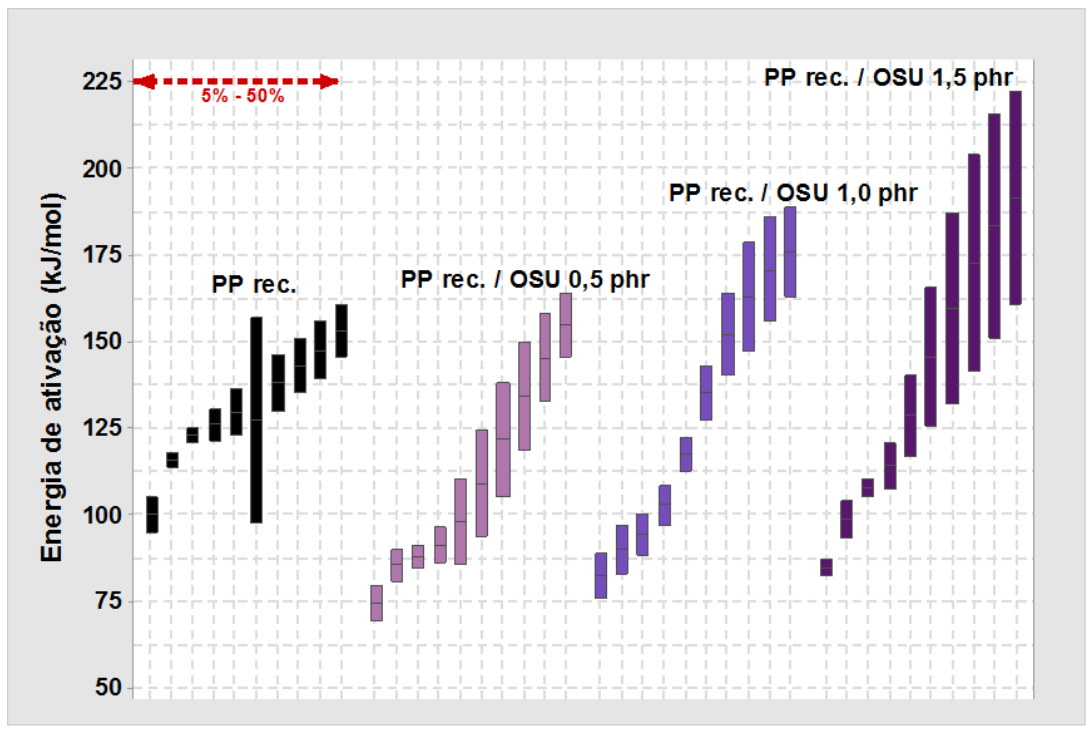

Figura 2: Valores médios e desvio-padrão para a energia de ativação, estimada pelo método OFW, para o intervalo de 5\% a 50\% de perda de massa. Amostras de PP rec. e PP rec. contendo diferentes teores de OSU.

Na Tabela 5, os dados de energia de ativação de cada amostra são sumarizados e o intervalo de confiança (IC) de $99 \%$ para o valor médio da energia de ativação do processo de degradação termo-oxidativa é exibido.

Pode ser observado pela Figura 2 que o modelo OFW afasta-se do ajuste linear dos dados experimentais, proposto pela Equação 1, conforme a amostra vai perdendo percentuais maiores de massa. De forma geral, 
em percentuais próximos de $50 \%$, há um aumento da dispersão dos valores de $\mathrm{E}_{\mathrm{a}}$ indicado pelo tamanho dos retângulos desenhados, especialmente no caso de PP rec./OSU 1,5 phr.

Além disso, a análise da Figura 1 e dos dados expostos na Tabela 5 permitem que a real influência do óleo de semente de uva possa começar a ser explicada. Observando o valor médio de energia de ativação para a degradação termo-oxidativa, assim como os respectivos desvios-padrões, das amostras de PP rec./OSU em relação ao PP rec. puro, OSU parece apresentar alguma ação antioxidante, quando incorporado ao PP rec, somente em teores mais elevados, ou seja, equivalente a 1,5 phr.

Tabela 5: Dados resumidos para o processo de degradação termo-oxidativa das amostras de PP rec. contendo diferentes teores de OSU

\begin{tabular}{ccccc}
\hline Amostra & $\begin{array}{c}\text { Energia de ativação } \\
\text { média }(\mathbf{k J} / \mathbf{m o l})\end{array}$ & $\begin{array}{c}\text { Desvio-padrão } \\
(\mathbf{k J} / \mathbf{m o l})\end{array}$ & $\begin{array}{c}\text { Erro padrão } \\
\text { da média } \\
(\mathbf{k J} / \mathbf{m o l})\end{array}$ & $\begin{array}{c}\text { IC de 99\% para } \boldsymbol{\mu} \\
\mathbf{( k J / m o l})\end{array}$ \\
\hline PP rec. & 130,4 & 17,9 & 3,3 & $121,4-139,4$ \\
\hline PP rec./OSU 0,5 $\mathbf{~ p h r}$ & 110,3 & 27,6 & 8,7 & $81,9-138,6$ \\
\hline PP rec./OSU 1,0 phr & 128,4 & 35,5 & 11,2 & $91,8-164,9$ \\
\hline PP rec./OSU 1,5 ph & 138,2 & 38,1 & 12,1 & $99,0-177,4$ \\
\hline
\end{tabular}

Tal consideração é o oposto ao discutido anteriormente quando da análise do diagrama de boxplot e demonstra a necessidade do uso das ferramentas estatísticas para melhor compreensão de resultados experimentais. O coeficiente de variação (CV), também conhecido como desvio padrão relativo (DPR), é uma medida padronizada de dispersão de uma distribuição de probabilidade ou de uma distribuição de frequências. Os CV's calculados para as composições de PP rec./OSU 0,5 phr, PP rec./OSU 1,0 phr e PP rec,/OSU 1,5 phr foram de 25,0\%,27,6\% e 27,6\%, respectivamente. Consequentemente, o que de fato ocorre está ocorrendo é que a maior imprecisão do modelo OFW, ao modelar os dados das composições de PP rec. com maior teor de OSU, acabou por induzir a uma falsa conclusão sobre a verdadeira ação do óleo vegetal.

A fim de averiguar mais profundamente a verdadeira ação de OSU, o procedimento denominado análise de variância (ANOVA) de um fator foi aplicado com um IC de 95\%. A normalidade dos dados experimentais foi assegurada segundo o teste Shapiro-Wilk $(p=0,035)$, porém a igualdade de variância foi violada $(p<$ 0,010). Por consequência, a análise foi levada adiante com o teste de Kruskal-Wallis por postos usando-se o método de Dunnett para comparações múltiplas com um padrão - a amostra de PP rec. foi selecionada.

Segundo a análise do relatório estatístico, as diferenças entre os valores de $\mathrm{E}_{\mathrm{a}}$ das amostras de PP rec. e PP rec./OSU foram estatisticamente significativas em um intervalo de confiança de 95\% $(H=11,05, p=$ 0,011). Porém, ao serem comparadas com a amostra padrão de PP rec., o método de Dunnett revelou que a amostra de PP rec./OSU 0,5 phr foi a única que efetivamente apresentou algum efeito diferenciado para a influência do OSU. Tal conclusão reforça o discutido preliminarmente e corrobora com as hipóteses levantadas a partir do diagrama boxplot (Figura 1) e da Tabela 4. A Figura 3 ilustra os resultados encontrados pela aplicação do método de Dunnet.

O intervalo de confiança de 99\%, exposto na Tabela 5, para o valor da energia de ativação do processo de degradação termo-oxidativa de cada amostra, em cada taxa de aquecimento, permitiu que as etapas 2 e 3 da metodologia tivessem prosseguimento. Após o peneiramento dos modelos cinéticos, descritos na Tabela 1, os mecanismos hipotéticos mais apropriados foram escolhidos para modelar a degradação de cada amostra, em cada taxa de aquecimento. Na Figura 4, os modelos cinéticos selecionados estão identificados pelos respectivos códigos. Também estão indicados os valores de $\ln \mathrm{A}_{\mathrm{inv}}$ e $\mathrm{E}_{\mathrm{inv}}$, estimados pelo método dos parâmetros cinéticos invariantes (IKP).

Na maioria dos polímeros a oxidação ocorre em reação através de radicais livres. É um processo de auto-oxidação que se caracteriza por dois aspectos: (i) autocatálise; e, (ii) pode ser inibida por componentes externos (aditivos). As reações básicas do processo oxidativo seguem a mesma teoria de oxidação de hidrocarbonetos de baixa massa molecular, compreendendo as etapas de iniciação, propagação, ramificação e terminação. Quanto aos antioxidantes, estes podem ser classificados em primários ou bloqueadores de cadeia, pois interrompem o ciclo de propagação, reagindo com os radicais $\mathrm{R}^{*}$ e ROO*, introduzindo novas reações de terminação; e, antioxidantes secundários, uma vez que destroem os hidroperóxidos, um dos principais iniciantes da oxidação. Os antioxidantes podem ainda atuar como retardadores de oxidação, reagindo mais lentamente com os radicais, introduzindo novas reações de propagação e prolongando a vida útil do material $[1,2]$.

A Figura 4 permite observar que as amostras de PP rec./OSU apresentam uma complexidade crescente 
em seus mecanismos hipotéticos de degradação termo-oxidativa, sobretudo quando maiores teores de OSU e taxas de aquecimento mais elevadas estão presentes. Tal complexidade se reflete, particularmente, nos valores dos parâmetros cinéticos $\mathrm{A}_{\text {inv }}$ e $\mathrm{E}_{\text {inv }}$ obtidos para PP rec./OSU 1,0 phr e PP rec./OSU 1,5 phr, os quais foram menos acurados - observe a diminuição dos coeficientes de regressão $r^{2}$.

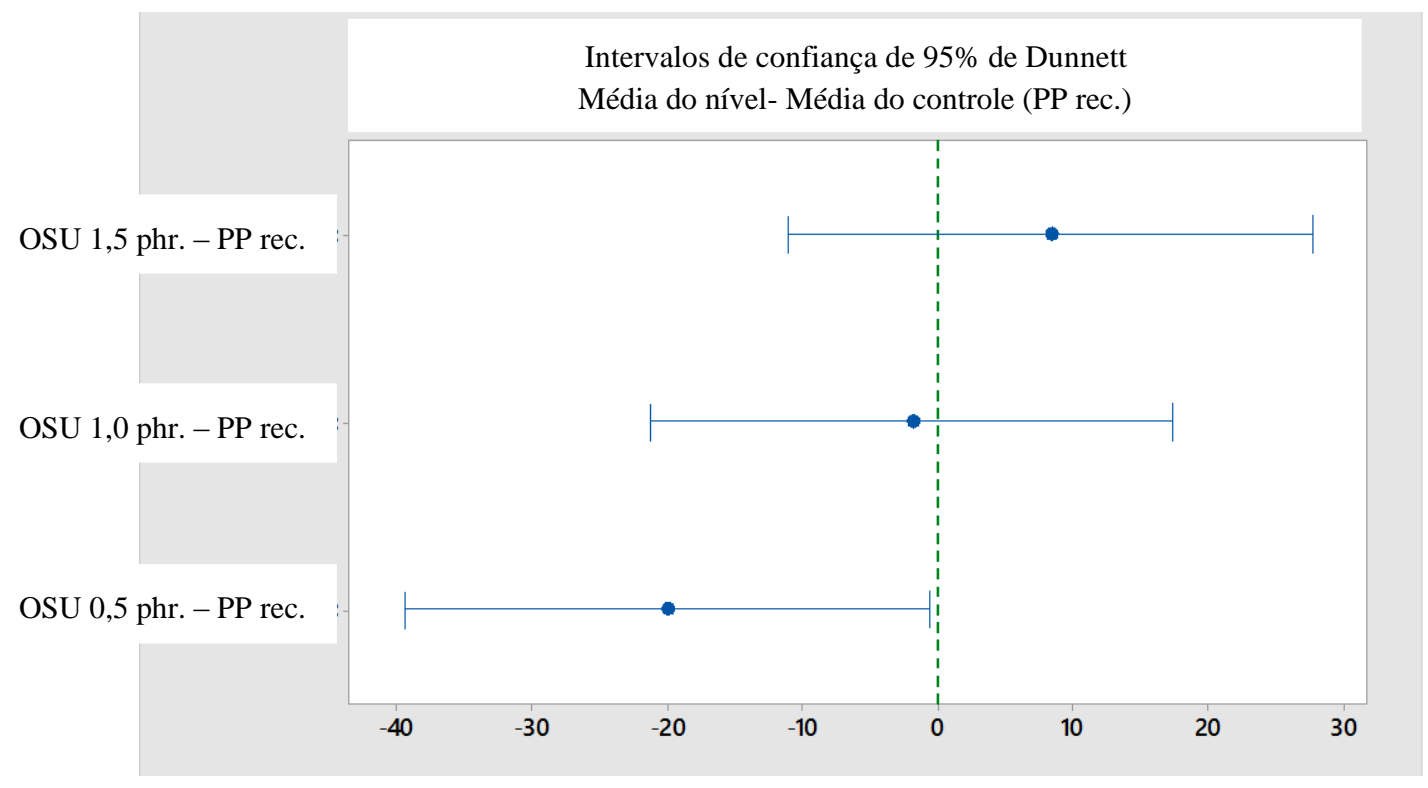

Figura 3: Resultados encontrados pela aplicação do método de Dunnet para o efeito do óleo de semente de uva sobre a energia de ativação do processo de degradação termo-oxidativa do PP rec. Se um intervalo não contiver o zero, a média correspondente será significativamente diferente da média de controle.

A Figura 4 permite também inferir que a presença de quantidades de OSU acima de 0,5 phr talvez introduza quantidades significativas de outros compostos químicos, além dos compostos fenólicos. Assim, a composição química complexa do óleo de semente de uva acabaria por propiciar o surgimento de reações laterais e/ou competitivas às reações de degradação já esperadas para o termoplástico, que OSU não se torna um antioxidante satisfatório. Um fator que ajuda a sustentar tal hipótese é o domínio de modelos do tipo $\mathrm{F}_{\mathrm{i}}$ (modelos lineares), cujas taxas de reação são constantes, na modelagem da degradação termo-oxidativa do PP rec./OSU 0,5 phr, fato que se opõe ao encontrado nas demais amostras, onde os modelos cinéticos mais intrincados do tipo $\mathrm{D}_{\mathrm{i}}$ e $\mathrm{A}_{\mathrm{i}}$ se tornam igualmente importantes na descrição do processo.

Portanto, a escolha de 0,5 phr como a quantidade mais promissora para a ação do óleo de semente de uva em uma eventual ação antioxidante, parece mais uma vez ser corroborada pela análise dos mecanismos hipotéticos previstos pela Figura 4.

\section{CONCLUSÕES}

Neste manuscrito, nosso grupo de pesquisa procurou determinar as condições ótimas para a aplicação do óleo de semente de uva como aditivo ao polipropileno reciclado. A análise termogravimétrica gerou dados experimentais sobre a degradação termo-oxidativa do termoplástico. Tais dados, tratados segundo a modelagem da cinética de degradação e análise estatística, permitiram chegar a duas conclusões fundamentais:

- O óleo de semente de uva (OSU), em função da sua composição química rica em compostos fenólicos, pode efetivamente atuar como um antioxidante natural para o polipropileno reciclado. Tal premissa permitiria especular sobre a substituição dos antioxidantes sintéticos costumeiramente usados;

- O potencial antioxidante de OSU é restrito a baixos teores de incorporação e a condições termooxidativas que se desenvolvem em baixas taxas de aquecimento. Quantidades maiores de OSU e/ou condições aceleradas de termo-oxidação causam efeito adverso.

\section{Agradecimentos}


Os autores agradecem aos órgãos de fomento FAPERJ, CAPES e CNPq, ao Programa Bolsa de Produtividade em Pesquisa 2019 da UNESA, e ao Laboratório de Biomateriais (IPRJ/UERJ).

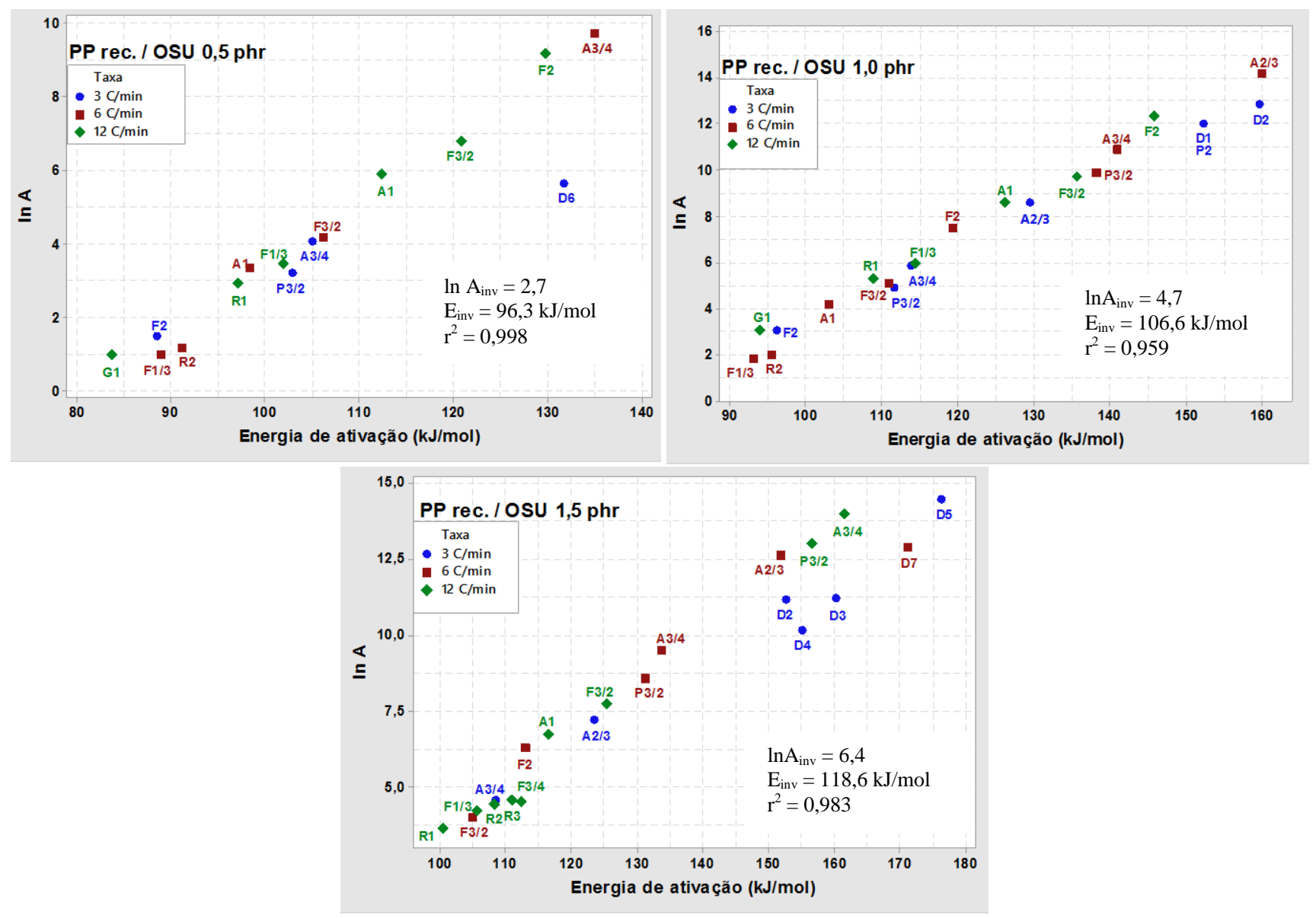

Figura 4: Modelos cinéticos hipotéticos para a descrição da degradação termo-oxidativa das amostras de PP rec./OSU segundo a metodologia empírica descrita no item 1.1. Os valores de $\mathrm{E}_{\text {inv }}$ e $\mathrm{A}_{\mathrm{inv}}$ foram determinados pelo método IKP.

\section{BIBLIOGRAFIA}

[1] DE PAOLI, M.A., Degradação e estabilização de polímeros, 2 ed, Chemkeys, versão online revisada, 2008.

[2] RABEllo, M., Aditivação de Polímeros, 1 ed, São Paulo, Artliber Editora Ltda, 2000.

[3] DELMONDES, P.H., FREIRE, J.M., SCHAEFER, A.S., et. al., "Substâncias antioxidantes presentes nos vegetais", Interdisciplinar: Revista Eletrônica da Univar, v. 9, n. 1, pp. 1-5, Jan. 2013.

[4] POMBO, J.C.P., RIBEIRO, E.R., PINTO, R.L., et. al., "Efeito antimicrobiano e sinergístico de óleos essenciais sobre bactérias contaminantes de alimentos", Segurança Alimentar e Nutricional, v. 25, n. 2, pp. 108-117, Maio/Agosto 2018.

[5] QUINARÍ, https://www.quinari.com.br, acessado em fevereiro de 2019.

[6] OLIVEIRA, R.R., LAGE, M.E., NETO, O.S., et al.“Antioxidantes naturais em produtos cárneos”, PUBVET, Londrina, v. 6, n. 10, ed. 197, art. 1324, 2012.

[7] FRITSCH, M., BOHN, A., KUHN, F., et al. "Caracterização da atividade antioxidante de frutas silvestres da região sul do Brasil”, In: XX Congresso Brasileiro de Engenharia Química, 1-7, Florianópolis, Santa Catarina, SC, Brasil, 19-22 de Out. 2014. 
[8] OLIVEIRA, K.A. “Obtenção de extratos padronizados em antioxidantes naturais: aproveitamento dos resíduos da Ucuúba (Virola surinamensis)”, Exame de Qualificação para Mestrado, Programa de PósGraduação em Ciências Farmacêuticas, Universidade Federal do Pará (UFPA), PA, 2018.

[9] ARVANITOYANNIS, I.S., LADAS, D., MAVROMATIS, A., "Potential uses and applications of treated wine waste: a review", International Journal of Food Science and Technology, v. 41, n. 5, pp. 475487, May 2006.

[10] OLIVEIRA, D.A, "Caracterização fitoquímica e biológica de extratos obtidos de bagaço de uva (Vitis Vinifera) das variedades Merlot e Syrah", Dissertação de Mestrado, Programa de Pós-Graduação em Engenharia de Alimentos, Universidade Federal de Santa Catarina (UFSC), SC, 2010.

[11] ZOPELLARO, S.R., SILVA, S.Z., LOVATO, S.R., “Compostos fenólicos totais e atividade antioxidante da farinha do resíduo da uva”, Journal of Health, v. 1, n. 2, pp. 154-163, 2019.

[12] ESTEVES, L.L., DA COSTA, H.M., RAMOS, V.D., et. al., "Cinética de degradação termooxidativa do polipropileno (PP). A determinação do mecanismo através da análise termogravimétrica (TGA/DTG)", In: XX Encontro Nacional de Modelagem Computacional (ENMC) e VIII Encontro de Ciência e Tecnologia de Materiais (ECTM), Nova Friburgo, RJ, 16 a 19 de outubro de 2017.

[13] ESTEVES, L.L., "A Influência de óleos vegetais sobre a degradação e propriedades do polipropileno reciclado", Dissertação de Mestrado, Programa de Pós-Graduação em Ciência e Tecnologia de Materiais, Instituto Politécnico (IPRJ), Universidade do Estado do Rio de Janeiro (UERJ), Nova Friburgo, RJ, 2018.

[14] COSTA, H.M., RAMOS, V.D., ESTEVES, L.L., et. al., "An empirical method for determination of kinetic models and kinetic parameters associated to thermo-oxidative degradation of recycled polypropylene (PP)", Journal of Engineering Research and Application (IJERA), v. 8, n. 9, pp. 15-27, Part 3, Sep. 2018.

[15] ACHILIAS, D., PANAYOTIDOU, E., ZUBURTIKUDIS, I., “Thermal degradation kinetics and isoconversional analysis of biodegradable poly(3-hydroxybutyrate)/organomodified montmorillonite nanocomposites", Thermochimica Acta, v. 514, n. 1-2, pp. 58-66, February 2011.

[16] TRACHE, D., ABDELAZIZ, A., SIOUANI, B., “A simple and linear isoconversional method to determine the pre-exponential factors and the mathematical reaction mechanism functions", Journal of Thermal Analysis and Calorimetry, v. 128, n. 1, pp. 335-348, April 2017.

[17] ERCEG, M., KREŠIĆ, I., VRADEČIĆ, N.S., et. al., "Different approaches to the kinetic analysis of thermal degradation of poly(ethylene oxide)", Journal of Thermal Analysis and Calorimetry, v. 131, n. 1, pp.325-334, January 2018.

[18] BOURBIGOT, S., FLAMBARD, X., DUQUESNE, S., "Thermal degradation of poly $(p$ phenylenebenzobisoxazole) and poly( $p$-phenylenediamine terephthalamide) fibres", Polymer International, v. 50, n. 1, pp.157-164, January 2001.

[19] CHRISSAFIS, K., "Kinetics of thermal degradation of polymers. Complementary use of isoconversional and model-fitting methods", Journal of Thermal Analysis and Calorimetry, v. 95, n. 1, pp. 273-283, January 2009.

[20] DAHIYA, J.B., KUMAR, K., MULLER-HAGEDORN, M., et. al., "Kinetics of isothermal and non-isothermal degradation of cellulose: model-based and model-free methods", Polymer International, v. 57, n. 5, pp. 722-729, May 2008.

[21] KHAWAN, A., FLANAGAN, D.R., "Solid-state kinetic models: basics and mathematical fundamentals", The Journal of Physical Chemistry B, v. 110, n. 35, pp. 17315-17328, August 2006.

\section{ORCID}

Helson Moreira da Costa

Valéria Dutra Ramos

Jacques Satler Andrade Estanislau Cyrino https://orcid.org/0000-0003-4916-7808

https://orcid.org/0000-0003-2263-2810

https://orcid.org/0000-0001-7569-990X 
